\title{
Creatividad, Aprendizaje Creativo y Desarrollo del Sujeto Creador PRIVADO
}

\section{Creativity, Creative Learning, and Developing the Creative Indi- vidual}

\author{
Alberto F. Labarrere Sarduy \\ Universidad Santo Tomás de Chile
}

(Recepción: octubre 2004 - Aceptación: noviembre 2004)

\begin{abstract}
En esferas de la actividad humana como la producción, el arte, la ciencia, los servicios, etc., el fomento de la creatividad ha adquirido relieve sin precedentes. El aumento de la potencialidad creativa de los individuos, los grupos, las organizaciones e incluso las poblaciones, se ha constituido en un objetivo consustancial del progreso y desarrollo de la sociedad y las personas. Los sistemas educacionales, por su lado, ponen cada vez más énfasis en lo que se denomina enseñanza y aprendizaje creativos, dirigen gran parte de sus esfuerzos hacia la búsqueda e implementación de métodos, programas y estrategias capaces de actuar sobre la potencialidad creadora de estudiantes y profesores. Resulta significativa la cantidad de programas de enseñanza que hacen del desarrollo de la creatividad uno de sus objetivos centrales.

A partir de la experiencia práctica e investigaciones ejecutadas por el autor, el presente artículo realiza una aproximación a la creatividad destacando algunas de las principales ampliaciones conceptuales que se han dado en este terreno y reflexiona en torno a las vías para promover el desarrollo del sujeto creador en la enseñanza.

Palabras claves: Creatividad, aprendizaje creativo, sujeto creador.
\end{abstract}

In fields of human activity such as production, art, science, services, etc., fostering creativity has reached unprecedented levels of importance. Increasing the creative potential of individuals, groups, organizations, and even of populations has become a consubstantial goal of societal and individual progress and development. Educational systems are placing ever more emphasis on what is termed creative teaching and learning and channel a major part of their efforts towards searching out and implementing methods, programs, and strategies that can increase the creative potential of students and teachers. A significant number of teaching programs have as one of their main objectives that of developing creativity.

Based on the author's research and practical experience, this paper approaches creativity by highlighting some of the primary conceptual advances that have arisen in this area and considers ways to help shape creative individuals in education.

Key words: Creativity, creative learning, creative individual.

En esferas de la actividad humana como la producción, el arte, la ciencia, los servicios, etc., el fomento de la creatividad ha adquirido relieve sin precedentes. El aumento de la potencialidad creativa de los individuos, los grupos, las organizaciones e incluso las poblaciones, se ha constituido en un objetivo consustancial del progreso y desarrollo de la sociedad y las personas.

Los sistemas educacionales, por su lado, ponen cada vez más énfasis en lo que se denomina enseñanza y aprendizaje creativos, dirigen gran parte de sus esfuerzos hacia la búsqueda e implementación de métodos, programas y estrategias capaces de actuar sobre la potencialidad creadora de estudiantes y profesores. Resulta significativa la cantidad de programas de enseñanza que hacen del desarrollo de la creatividad uno de sus objetivos centrales.
Múltiples son los tópicos susceptibles de ser debatidos respecto a las vías por las cuales la enseñanza y el aprendizaje pueden tornarse creativos. Por ejemplo, convendría cuestionarse si modelos de la creatividad y su desarrollo, directamente importados del quehacer científico y, por tanto, válidos para esta actividad, resultan igualmente eficientes para caracterizar la creatividad de los estudiantes y orientar los intentos de promoverla.

No menos importante podría ser interrogarnos acerca de si el simple aprendizaje de técnicas para la creación y la solución creativa de problemas por los estudiantes, es suficiente para convertirlos en sujetos con una alta capacidad y disposición para crear. Igualmente resulta oportuno cuestionarnos respecto al papel del contexto en el desarrollo de la potencialidad creativa 
de los estudiantes y, finalmente, una pregunta de obligatoria formulación, se refiere al papel que desempeña el profesor en la educación de la creatividad de sus estudiantes; o sea, las relaciones entre la creatividad pedagógica (las habilidades, conocimientos, etc., del profesor creativo) y el aprendizaje creativo.

\section{Concepto y enfoques}

Creatividad por lo común es producción de novedad. Prácticamente todos coinciden en que ella interviene allí donde la actividad del ser humano, orientada por finalidades específicas, da lugar a objetos o productos originales según la época, el lugar, las circunstancias y, en suma, los patrones sociales y culturales por los que se juzga.

En su prólogo a su «Estructuras de la Mente», H. Gardner identifica tres nodos de los que surge la creatividad: «el individuo con su propio perfil de capacidades y valores; los ámbitos para estudiar y dominar algo que existen en una cultura; $y$ los juicios emitidos por el campo que se considera como competente dentro de una cultura (Gardner, 1995, pág. 16). A renglón seguido define al individuo creativo como «quien resuelve regularmente problemas o inventa productos en un ámbito, y cuyo trabajo es considerado innovador y aceptable por los miembros reconocidos de un campo (op. cit., pág. 16).

Coincidiendo con Gardner, Mitjans expresa que la creatividad «es el proceso de descubrimiento o producción de 〈algo nuevo〉 que cumple exigencias de una determinada situación social, en el cual se expresa el vínculo de los aspectos cognitivos y afectivos de la personalidad» (Mitjans, 1989, pág. 39).

Por su parte De la Torre $(1993,1996)$, ha planteado que al representárnosla debemos tener en cuenta cuatro factores: el producto, la persona, el proceso y el medio o ambiente.

Quien trate de asomarse a la creatividad hoy en día, no puede hacerlo dejando a un lado su naturaleza compleja, manifiesta en la interdependencia de los diversos factores a través de los cuales ella se expresa. Por tal razón, si hasta hace algún tiempo podía aceptarse el énfasis único en los procesos cognitivos e instrumentales intervinientes, ahora es requisito abordar el tópico con enfoques más integrales. Procesos como la constitución de los juicios valorativos y los contextos socioculturales, relativamente marginados del universo de los fenómenos creativos en estudio, emergen ahora con una fuerza considerable en la investigación.

Todo apunta a la consideración de la creatividad como un fenómeno sumamente complejo, en vista de lo cual únicamente una aproximación igualmente compleja y multidimensional podría dar cuentas de sus mecanismos, así como de las condiciones y vías para su desarrollo en cualquier sujeto y contexto. En el de la educación la dificultad se hace mayor debido a la presencia de factores asociados con la actividad formativa: las habilidades del profesor, la naturaleza de los currículos, los sistemas evaluativos y otros.

\section{Ampliaciones conceptuales}

Al parecer el concepto de creatividad se está ampliando por la incorporación de nuevas exigencias definitorias, aunque se mantiene la producción de novedad $\mathrm{u}$ originalidad como esencial. Un ejemplo lo encontramos en el propio De la Torre, quien ha llegado a expresar que la creatividad debe considerarse como la «... capacidad y actitud para generar ideas nuevas y comunicarlas» (De la Torre, 1996; énfasis nuestro). Este mismo autor en una aproximación anterior ya había introducido un aspecto de suma importancia, al atribuirle la característica de ser una actitud comprometida con uno mismo y con los demás (De la Torre, 1996).

En las dos aproximaciones de De la Torre, resulta interesante la introducción de la capacidad para comunicar las ideas (el producto) y la noción de compromiso. La referencia a que no basta generar algo novedoso, sino que es necesario su expresión en el acto comunicativo que presupone la interacción con el otro, puede ser tomada como reconocimiento del factor social: quien crea algo debe comunicarlo de alguna manera a los otros, debe tener la posibilidad de ponerlo en contacto con el producto de su actividad y actuar sobre los patrones de aceptación y emisión de juicios valorativos, por los cuales el producto se considera novedoso o no. La dimensión comunicativa de la creatividad puede asumirse como expresión del carácter activo del sujeto.

La segunda idea, el carácter comprometido de la creatividad, nos aproxima a que toda producción relevante, significa un acto de responsabilidad del creador, e incluso de quienes valoran a él y su producto como creativos. Hay ejemplos suficientes de cómo una actitud con 
un bajo compromiso social puede conducir a producciones muy novedosas y a la vez perjudiciales para la humanidad.

De forma similar deberían explorarse otros ángulos de la definición clásica; por ejemplo, el referido a la utilidad del producto y su valoración por la comunidad, podría conducirnos a criterios mucho más específicos para determinar lo que se considera o no creativo respecto a una actividad determinada, obteniéndose por esta vía mayor no sólo precisión en la definición sino criterios de valoración más adecuados para juzgar qué se considera o no creativo.

A partir de lo dicho, es apropiado argumentar que en el ámbito pedagógico, la creatividad debería caracterizarse no sólo mediante los parámetros habituales y expresados de manera general; sino además, por otros relevantes (en el sentido de deseabilidad normativa) para los cometidos de la enseñanza y el aprendizaje.

Una tentativa en esta dirección es la acometida por Martínez (2000), con fundamento en nuestra concepción de aprendizaje y enseñanza para el desarrollo (Labarrere, 1999; 2000). La autora considera la creatividad pedagógica no únicamente como producción novedosa, sino por su expresión en procesos de orientación directa, consciente y reflexiva hacia el desarrollo creativo del estudiante o del propio profesor.

Tal comprensión de la creatividad del profesor, se sustenta en el convencimiento de que para el contexto pedagógico la finalidad principal es generar procesos conducentes al desarrollo de los estudiantes y de sí mismo, en vista de lo cual todo proceso o actividad genuinamente pedagógica, debe orientarse hacia tal cometido, no espontáneamente sino de manera consciente y reflexiva.

La dificultad que se genera entonces, reside en identificar cuándo la creatividad está orientada por motivaciones de desarrollo y cuándo no es así. En este sentido acudimos al reporte del sujeto y analizamos su expresión verbal en la clase o en sesiones especialmente concebidas. Los análisis de la reflexión de profesores (Quintanilla, Labarrere y Araya, 2000; Martínez, 2000), han permitido constatar que en muchas ocasiones cuando los profesores elaboran recursos didácticos, los motivos que los animan consisten en "crear, dar vida al recurso» y no en desarrollar la creatividad del estudiante. En sus reflexiones predominan las referencias a las exigencias tecnológicas del medio que están elaborando, ubicándose su pensamiento en lo que hemos denominado plano instrumental operativo (Labarrere, 2000). En este plano los problemas que se resuelven conciernen más directamente a los objetos y recursos didácticos que al desarrollo de los estudiantes.

La educación de la creatividad, enfocada desde el ángulo de su orientación al desarro1lo, permite también precisar las modificaciones que experimenta la actividad de los profesores. Por ejemplo, los momentos en que la valoración de los actos creativos no orientada hacia el desarrollo comienza a estar dirigida por motivos de esta naturaleza; y lo contrario, momentos en que el profesor «desconecta» las motivaciones de desarrollo de la creatividad del estudiante y pone en primer plano la confección de un recurso por sí mismo.

En lo concerniente al aprendizaje creativo, expresamos algo similar; o sea, que un elemento básico del desarrollo de la creatividad del estudiante, radica en su expresión mediante procesos y actos de reflexión, toma de conciencia y autoconocimiento de la propia creatividad y la ajena (la de sus compañeros y la del profesor), a la vez que los motivos y orientaciones principales son los dirigidos hacia el desarrollo y el aprendizaje creativo (Labarrere, 2000; Quintanilla y Labarrere, 2000).

El compromiso, que a decir de De la Torre entraña la creatividad, nosotros lo hemos enfocado a través del desarrollo de la responsabilidad en la creación como característica a formar en el sujeto creador (Labarrere, 1999; 2000). En este contexto la responsabilidad se expresa no sólo en o ante el producto creado; sino sobre todo en el compromiso del sujeto en los mismos procesos de desarrollo de la creatividad propia y de los compañeros, lo cual sitúa nuevas exigencias para los procesos formativos $y$ confiere a los participantes posiciones y roles diferentes de los habitualmente reconocidos.

\section{Potencialidad para crear}

En muchas áreas del funcionamiento humano suele establecerse diferenciación entre potencialidad y realización. La creatividad no es un caso distinto. La lógica para la división se pierde en el tiempo. Recordemos, por ejemplo, cómo en las décadas de los cincuenta y sesenta, en el dominio del aprendizaje, a la vera de la 


\section{LABARRERE}

polémica entre los promotores del enfoque asociacionista estímulo respuesta y los cognitivistas, la evidencia del aprendizaje sin ejecución asestó un fuerte golpe a los partidarios de la conexión entre estímulos y respuestas. La evidencia aportada fue la posibilidad de aprendizaje sin ejecución. Un grupo de investigadores de tradición cognitivista mostró casos donde los individuos podían haber aprendido algo y por diversas razones no lo ponían en práctica. El hecho, contemplado de cierta manera, puede ser asumido como una muestra de potencialidad no realizada.

Otra ilustración de la diferenciación entre potencialidad y realización fue suministrada por Vigotsky con su noción de zona de desarrollo próximo. En referencia al desarrollo intelectual, este genio de la Psicología dejó en claro que no todos los sujetos manifiestan igual nivel de desarrollo potencial, cuando se considera lo que pueden hacer por sí solos (desarrollo actual) y lo que les resulta accesible mediante ayuda o cooperación.

Respecto a los planteamientos de Vigotsky, acerca de la naturaleza de lo potencial pueden darse variadas interpretaciones (Corral, 1999, Labarrere, 1999, 2000). Una consiste en asumir que ella existe en el sujeto, considerándola como propiedad interpersonal y otra que la asume cual peculiaridad no privativa del sujeto; sino en calidad de construcción que tiene lugar a partir de procesos interactivos del sujeto con diversos exponentes de la sociedad y la cultura.

Para algunos autores, la ejecución independiente que sigue a procesos de ayuda o colaboración en la zona de desarrollo próximo, debe entenderse como creación individual que expresa la actualización de la potencialidad. Son conocidas otras interpretaciones igualmente inclinadas al reconocimiento de la potencialidad. Por ejemplo, la del aprendizaje mediado de Feuerstein (1980). Este autor, recordando en mucho a Vigotsky, asume que toda persona dentro de los parámetros de normalidad evidencia potencialidad para lograr niveles más altos de desarrollo cognitivo, si ocurren actos de mediación por instrumentos culturales.

Otros autores, como por ejemplo Mitjans (1996), o el propio Feuerstein, incluso han argumentado la imposibilidad de negar la existencia de creatividad en personas con déficit intelectual y plantean que con un programa y criterios adecuados, es posible el despliegue de la potencialidad creativa en ellas.
Desde nuestro punto de vista, la potencialidad para crear está presente en toda persona. Lo mismo que Mitjans y otros autores, consideramos actividad y expresión creativa en sujetos con deficiencias intelectuales que les permitan mantener un nivel adecuado de actividad simbólica. De manera aún más radical, decimos que toda persona, que cumpla el requisito anterior y haya completado los estadios "pertinentes" del desarrollo, puede haber intervenido en procesos de producción creativa, diferentes por su naturaleza, grado y significación.

En la constitución de la potencialidad para crear concurren diversos elementos: los conocimientos sobre el área particular que posea el sujeto, los instrumentos para interactuar a su alcance y, desde luego, de sus motivaciones e intenciones. De hecho la potencialidad se expresa en forma compleja y contradictoria; se constituye y modifica dinámicamente, en dependencia de factores y peculiaridades pertenecientes a la persona y la trama social.

De acuerdo a lo anterior, resulta sin fundamento afirmar que un sujeto, que cumple el requisito de normalidad, carece de potencialidad para crear en uno $\mathrm{u}$ otro ámbito de la actividad; lo que ocurre es que esta potencialidad está asociada a diversos factores, unos que la facilitan y otros que la entorpecen. Los factores que actúan sobre la potencialidad creadora pueden variar por su fuente (el propio sujeto o el contexto sociocultural donde actúa). Mitjans (1989, 1996), desde su concepción personológica y configuracional ha considerado la expresión creativa como dependiente de la implicación del sujeto en la actividad, trayendo a primer plano los factores motivacionales. Aun cuando respecto a lo planteado no coincidimos totalmente con la autora, por razones que no vamos a abordar aquí, resulta indiscutible que la ausencia' de motivación es un elemento que debe ser tenido en consideración.

Al carecer de sentido afirmar que una persona no es potencialmente apta para crear, puede comprenderse que cuando la potencialidad no se explicita, se debe a la acción de uno o varios factores de los antes señalados, que han ejercido un efecto entorpecedor; las contingencias acompañantes de la actividad, el contexto o el ambiente, pueden desempeñar un papel importante en este sentido.

Es cierto que la potencialidad para crear ha sido interpretada sobre todo como una propie- 


\section{CREATIVIDAD, APRENDIZAJE CREATIVO Y DESARROLLO DEL SUJETO ...}

dad del sujeto. Ante esta situación parece razonable elaborar una representación del sujeto potencialmente creador, capaz de recubrirlo explícitamente no sólo a él, sino extendida hasta abarcar la situación en que se halla (el contexto). Con este entendimiento, hablaríamos de la potencialidad teniendo en mente más la interacción que el hecho individual. Tal proceder ayudaría a comprender la potencialidad como construcción mediante la interacción y daría cuentas de la razón por la cual los sujetos muestren diferente niveles de potencialidad en contextos diversos, ante influencias ambientales diferentes.

Por otro lado, la potencialidad no puede verse como patrimonio de los individuos aislados, en nuestra opinión debe considerársele como una peculiaridad también de los grupos, las organizaciones, etc., sometidos a criterios como los anteriormente señalados. De manera similar a como es un imperativo reconocer la diversidad creativa de las personas, de los grupos y hasta de las poblaciones, no menos importante resulta en el caso de la potencialidad para crear. Esta concepción está en la base de intentos de educación de la creatividad y desarrollo del sujeto cuyo énfasis recae sobre el desarrollo creativo no de los individuos aislados, sino de grupos amplios. A partir de lo dicho se hace clara la necesidad de considerar la expresión de la subjetividad creativa no sólo a nivel individual, sino también como un fenómeno de naturaleza grupal o colectiva.

\section{Solución de problemas y creatividad}

Todo acto creativo, en algunos de sus momentos, se manifiesta como formulación y solución de problemas, estos procesos constituyen el contenido y el medio, por excelencia, donde ocurren los principales actos creativos del hombre En tal sentido muchos estudiosos, encabezados los partidarios de la escuela Gestalt (Kofka, Dunker, Maier y otros), mostraron que a la solución de verdaderos problemas le resulta consustancial la ocurrencia de reestructuraciones en el contenido de los mismos y, a la vez, en el pensamiento de los individuos; insertos en procesos reestructuradores de diversa naturaleza, los objetos, fenómenos y hasta las mismas relaciones adquieren los nuevos significados que identifican al quehacer creativo.

Para el sujeto creador es sumamente importante ver el problema (o los problemas) desde diferentes perspectivas; colocarse él mismo y colocar el problema en posiciones o contextos relacionales diversos, desde los cuales se revelan nuevas propiedades. Este mecanismo, por el cual, como hace tiempo enfatizara Rubinstein (1986), surgen y se transforman los significados, ha sido asumido explícita o implícitamente, como de núcleo de los procesos y actos creativos y, consecuentemente, objetivo central del desarrollo de la creatividad.

Por la razón aludida, uno de los aspectos más trabajados en lo concerniente al desarrollo de la creatividad en la escuela, es el aprendizaje de técnicas para solucionar creativamente problemas y desarrollar el pensamiento. Cuando se analiza la introducción de estas técnicas en la escuela, puede observarse el intento por ofrecer medios para que el estudiante se habitúe y sea capaz de ver la situación (el problema) desde diferentes ángulos, a fin de que le sea posible extraer (formar, construir) diversos significados para uno $\mathrm{u}$ otro de sus componentes y el proceso de solución como un todo. En tal sentido, resulta criticable la manera de hacer, que permanece prácticamente inalterable respecto a la predominante en otras áreas del quehacer humano.

Cuando asumimos la solución de un verdadero problema como acto creador, diferenciamos uno de sus momentos como núcleo del desarrollo de la creatividad en el contexto pedagógico: aquel en que el sujeto, ya dentro de ese espacio de producción creativa donde los recursos (conocimientos, estrategias, etc.) a la mano se han demostrado insuficientes para darle acceso directo e inmediato a la solución deseada, toma conciencia de ello, no abandona la búsqueda y continúa realizando esfuerzos en tal sentido. Así, para el desarrollo de la creatividad, y sobre todo del sujeto creador, no son cualquier tipo de reestructuraciones (en los objetos, el contexto y el propio sujeto), sino precisamente las que tienen lugar en ese espacio de limitación, perseverancia y conciencia, las que resultan cardinales y, como afirmamos, constituyen el eje del desarrollo y educación del sujeto creador.

En la tríada limitación-perseverancia-conciencia, se expresan tres factores principales para la formación del sujeto. En primer lugar, el paso hacia la zona de creatividad que se produce en toda solución de un verdadero problema, cuando tiene lugar ese colapso de «lo habitual», y el sujeto "salta» hacia el espacio donde predomina la incertidumbre y el desequilibrio que han 
sido considerados habitualmente como peculiares del proceso creativo. En segundo orden, la permanencia del sujeto en ese espacio, así como la asunción del reto que entraña generar nuevas y sucesivas opciones de solución. Por último, la conciencia del proceso protagonizado y la subjetivización del actuar creativo.

Para el desarrollo de la creatividad en los contextos pedagógicos es necesario tener en consideración los tres elementos mencionados. Respecto a la conciencia, puede señalarse que comúnmente los intentos de actuar sobre la creatividad la han considerado como toma de conciencia acerca de los instrumentos empleados para la creación, lo cual resulta comprobable mediante la revisión detenida de los programas para facilitar la invención, la fantasía y la solución reportados en la literatura. La conciencia y el conocimiento del sujeto, acerca de su inserción en la situación creativa, han sido tratados sólo muy parcialmente.

Que la acción pedagógica, o didáctica, acepte como eje de su influencia la inserción del estudiante en el espacio de producción creativa durante la solución de problemas, es sólo promesa o acaso premisa del desarrollo creativo, pero no garantiza, sin más, que éste tenga lugar. $\mathrm{Al}$ respecto debe considerarse la manera y condiciones bajo las cuales él se inserta en dicho espacio, toma conciencia de las limitaciones y la intencionalidad o intencionalidades que lo movilizan; es decir, la subjetividad en sintonía con los requerimientos inherentes a la actividad creadora.

Más adelante, al abordar el desarrollo del sujeto creativo, nos ocuparemos en detalle del tópico, pero por ahora quisiera señalar, por sus implicaciones teóricas, que el desempeño del sujeto en el espacio de producción creativa -igual que en la solución como un todo-, no puede comprenderse como un hecho de transformación cognitiva instrumental solamente; sino ser enfocado como suceso con profundas connotaciones afectivo emocionales, valorativas y axiológicas; cuyas repercusiones se extienden hasta abarcar la capacidad «instrumental» del sujeto, su disposición y postura personal frente a la (su) creatividad y los productos a que da lugar.

No debe concluir esta sección, sin abordar la formulación de problemas como expresión de la creatividad. Prácticamente todos los autores coinciden en que la creatividad se expresa también como formulación de problemas; para muchos el sujeto creador sobre todo genera pre- guntas, descubre problemas en situaciones para otros triviales. De hecho, gran parte de los intentos por educar la creatividad se preocupan por desarrollar la capacidad de encontrar y formular problemas. Nosotros mismos hemos trabajado en esta dirección. Nuestro punto de vista asume que solución y producción de problemas son actividades complementarias en su repercusión práctica, pero presentan elementos y exigencias comunes para el sujeto. Incluso llegamos a afirmar que la formulación de problemas puede verse como un tipo especial de solución, por lo cual prácticamente todo lo expresado respecto a esta última resulta válido para la primera.

\section{La experiencia creativa}

Es reconocido que en el contexto pedagógico los productos creativos a que arriba el estudiante por lo común presentan sólo novedad relativa. Por ésta se entiende la peculiaridad de ser nuevos únicamente para sus creadores y/o para todos o algunos de sus compañeros. Si entendida desde las peculiaridades definitorias básicas (novedad, originalidad, etc.) la creatividad es una convención del quehacer pedagógico, no resulta lo mismo para los procesos subyacentes que muy poco, o en nada se diferencian de los que tienen lugar en el científico, el artista y otras personas.

En la ciencia y otras áreas del conocimiento y actividades sociales, por el contrario, un producto nuevo para su creador debe serlo también para la comunidad científica en el seno de la cual se produce; cuando esto ocurre es que se le considera creativo. Lo señalado evidencia la orientación hacia el producto y el proceso de producción, que resultan relevantes para la ciencia y otra esferas de la actividad humana y que, como ya expresamos, predomina también en la enseñanza. La novedad, así enfocada, está expuesta al criterio del valor. Por ejemplo, en la producción artística, el producto debe someterse a otros criterios valorativos, como los estéti$\cos$. En esta esfera un producto puede ser original, pero si no responde a los patrones estéticos reconocidos no se asume como tributo de la creatividad artística. Esto es una muestra de cómo la valoración social, antes aludida, condiciona «desde dentro» lo que ha de asumirse como creatividad.

En la educación, la novedad es relativa en la medida en que interesa más su proyección sub- 


\section{CREATIVIDAD, APRENDIZAJE CREATIVO Y DESARROLLO DEL SUJETO ...}

jetiva. De hecho, en este terreno, la acción sobre la creatividad desplaza su interés hacia los procesos; sin relegar o desconocer el producto, le resultan más relevantes los procesos por los que se obtiene; y más aún los componentes cognitivos, afectivos, relacionales, subyacentes a los procesos instrumentales, que estos últimos por sí mismos.

Lo anterior implica que a la enseñanza y el aprendizaje creativos, considerados desde la solución de problemas, le resulta relativamente más importante la inclusión y la proyección subjetiva del estudiante, la manera en que se relaciona con las situaciones en y desde las cuales crea, la disposición para creary los criterios por los que juzga acerca de la creación misma y su posición respecto a esta última.

Es indiscutible que la educación de la creatividad en los estudiantes no puede desconocer el valor y la función que desempeñan los conocimientos sobre el campo de que se trate y el dominio de los recursos para la solución de problemas; mas estos no deben ser analizados en sí y por sí mismos, sino a través del entramado que conforma la subjetividad. Como veremos más adelante, cuando este aspecto no se tiene suficientemente en consideración, la formación del sujeto creador puede verse entorpecida. Pero, ¿cómo se manifiesta esta inclusión? Nosotros hemos pensado que es bajo la forma de experiencia creativa.

La experiencia creativa es uno de los ejes principales del desarrollo creativo en la enseñanza; ella se manifiesta como el sentimiento de estar participando en una actividad mediante la cual se produce novedad y resulta accesible mediante la reflexión y el discurso del sujeto.

La experiencia creativa suele estar asociada a dos hechos: la participación en la actividad que está teniendo lugar y/o la vivencia de arribar a un producto novedoso. Estas dos circunstancias no excluyentes, adquieren diferente significación para el desarrollo creativo del sujeto. Cualitativamente la primera (estar incluido en la actividad creativa como un todo) es central.

No es lo mismo si el estudiante enfrenta la creación con el imperativo de obtener un producto novedoso, a si lo hace buscando participar en la actividad creativa; o más, en una situación desarrolladora de la creatividad. Cuando el estudiante opera en un contexto creativo persiguiendo un producto, la experiencia creativa suele tomar la forma de éxito o de fracaso, coincidentes con la obtención o no de ese producto.

Por lo común dicha experiencia se identifica con el éxito, pero no es exactamente lo mismo; el éxito puede ser visto como la obtención del resultado previsto, la experiencia creativa es la sensación de haber producido o estar produciendo novedad. Es precisamente con apoyo en lo último, que el estudiante procura solucionar problemas o generar objetos con la motivación de inclusión en una actividad de desarrollo.

En los intentos habituales de desarrollo de la creatividad, la experiencia creativa suele manifestarse inicialmente asociada a los productos o resultados finales; pero permanece anclada en este componente de la solución. En nuestras investigaciones hemos constatado que es posible y deseable que la experiencia de creación se desplace o amplíe hasta abarcar el proceso y las relaciones de los sujetos, de manera que sea gratificante y motivador no sólo arribar al objeto, a la solución deseada; sino la propia actividad de búsqueda y participación. Con esto se abre un nuevo y más amplio significado para la creatividad: el tránsito de su significado como encuentro de lo novedoso hacia el significado como búsqueda, intento y participación.

La relevancia de la experiencia creativa se establece a través del significado de la actividad del sujeto. En ocasiones los estudiantes no alcanzan la solución de un problema y, sin embargo, la experiencia creativa no se presenta como un fracaso. Estos son los casos en que los estudiantes se implican en determinada actividad y los motivos predominantes se corresponden con la participación en sí misma. Los estudiantes resultan reconocidos no por haber arribado a la respuesta correcta o por haber confeccionado el objeto más original, sino por haber intentado producir novedad y hacer cosas originales.

En investigaciones realizadas en el terreno de la solución de problemas matemáticos (Labarrere, 1999; Jiménez, 2000), se ha podido constatar el efecto favorecedor del desplazamiento de los criterios por los cuales se valora la creatividad (desde los resultados hacia la valoración del intento de ser creativo), sobre la formación y transformación de las creencias personales de los estudiantes; así como respecto a sus posibilidades para formular problemas originales y para hallar múltiples soluciones creativas a problemas de creación. 
Al considerar la experiencia creativa, es necesario tener en cuenta que ella ocurre en determinado contexto y en la relación con otras personas (estudiantes, profesores, etc.) que la legitiman a través de sus juicios sobre el producto y el desempeño que ha seguido el sujeto para su obtención. Por tanto, la experiencia creativa no se limita al individuo, sino que se extiende al grupo y aparece no sólo mediante la creación colectiva de determinado producto, sino a través de actos valorativos que involucran a varios sujetos.

\section{El sujeto creador}

En alguna ocasión A. A. Smirnov, hizo una brillante aproximación teórica cuyas repercusiones metodológicas y prácticas son indiscutibles. En referencia al estudio de los procesos psicológicos humanos, particularmente los cognitivos llamó la atención acerca de que es el hombre quien memoriza y no la memoria; es el hombre quien piensa y no el pensamiento, $\mathrm{y}$ así por el estilo.

Con esta simple y aguda reflexión, estaba arremetiendo contra la tradición que procura esclarecer la naturaleza de los fenómenos psíquicos, poniendo énfasis en el proceso por sí mismo, buscando entender sus mecanismos íntimos, sin tener en cuenta que dicho proceso se expresa, únicamente como una propiedad de la persona (el ser humano), con toda su carga de motivos, sentimientos, afectos y necesidades; desde una postura y posición personal irrepetibles.

Con la misma lógica en mente, habría que decir que crea el hombre y no la creatividad. En la actualidad la enseñanza que emana de la reflexión de Smirnov es compartida por muchos estudiosos y se materializa en el intento por comprender los hechos de la actividad creativa, y otras tantas, considerándolos desde enfoques más holísticos o integrales, que destacan el carácter sociocultural, personológico, etc.

En particular el enfoque personológico (González, 1997) incluye la categoría configuración para señalar la «... constitución subjetiva de los distintos tipos de relaciones y actividades que caracterizan la vida social de la persona ... categorías complejas, pluridimensionales, que representan la unidad dinámica sobre las que se definen los diferentes sentidos subjetivos de los eventos sociales vividos por el hombre» (González Rey,
1997, pág. 92). Según el autor esta categoría capta el carácter complejo, individual, irregular y contradictorio con la que se presenta la organización de la personalidad y, en ella, la dinámica de lo interno y lo externo, así como lo afectivo y lo cognitivo.

Dentro de la misma línea, pero en referencia específica a la creatividad (Mitjans, 1996), plantea que en el individuo ésta adopta la forma de configuraciones creativas, a las que atribuye la peculiaridad de expresar «la integración dinámica de los elementos personológicos que intervienen en la expresión creativa del sujeto» (pág. 28). Según la autora, asociados a la creatividad se encuentran un conjunto de elementos psicológicos, entre los cuales menciona la motivación, la autovaloración adecuada, la reflexividad y la elaboración personalizadas, la capacidad para tomar decisiones, el planteamiento de metas y la capacidad volitiva para la orientación intencional del comportamiento.

Con independencia del cuestionamiento acerca de la naturaleza indiferenciada de los elementos mencionados, posiblemente valederos no sólo para la creatividad sino también para otros procesos, la aproximación de Mitjans introduce elementos valiosos para la educación de la creatividad. Uno de ellos es el sujeto, que retoma de González Rey.

La categoría sujeto todavía no ha cerrado su ciclo de legitimación, sigue siendo sumamente polémica, plurisémica y contradictoria. Para los autores citados, el sujeto, relativamente considerado como diferente de la personalidad, toma sobre sí la responsabilidad por el comportamiento en momentos y circunstancias específicas de la vida cotidiana; se le reconoce la peculiaridad de ser «... el momento más activo e individualizado de la subjetividad humana (González Rey, 19996, pág. 86) y desde o a través de él se realiza la función de toma de decisiones y autorregulación intencional de los comportamientos.

La discusión puede comprender un conjunto muy amplio de aspectos, entre ellos los relativos a ¿cómo y cuándo se constituye el sujeto?, si ¿resulta o no accesible su formación o si es un proceso que escapa a la acción intencional y pedagógica?, ¿qué indicadores nos permiten juzgar respecto a los niveles de desarrollo? y otros muchos de naturaleza epistemológica y metodológica.

Pero suspendida la polémica, para nosotros la importancia de la categoría la vemos, pre- 
cisamente, en su referencia a la toma de decisiones, la regulación consciente e intencional del comportamiento $\mathrm{y}$, en general, hasta donde sea posible, de la interacción del individuo con el mundo. Es obvio que una concepción como la nuestra acerca de la creatividad, que enfatiza los momentos de reflexión y conciencia, resulta muy próxima a esta representación del sujeto. En nuestro criterio, no se trata únicamente del desarrollo de la creatividad, sino de la educación de un sujeto que desee y sea capaz de llevar sobre sus hombros la responsabilidad de su creatividad; comprendiendo por ello, incluso, la responsabilidad por su propia formación creativa. Lo que acabamos de afirmar puede parecer en extremo radical; pero de hecho, cuando hemos hablado de aprendizaje creativo, hacemos alusión a un proceso mediado por la responsabilidad del estudiante. Se trata de la constitución de una responsabilidad en aumento según se acrecienta el desarrollo del sujeto.

Desde luego, la referencia al sujeto no puede verse exclusivamente en su acepción individual, en sujeto se constituye también la colectividad de personas aunadas por intenciones y finalidades comunes, con una historia de desarrollo compartida y donde las relaciones características, de participación, ayuda y cooperación, pero también de oposición y conflicto, se tensan en función de la conservación y el desarrollo del grupo y de cada persona.

La idea que pretendemos recoger de los anteriores señalamientos, es que la educación de la creatividad, los intentos por la realización de la potencialidad creativa de los estudiantes, resultan escasamente fructíferas o truncas, si no aceptan para sí el desarrollo del sujeto creativo como objetivo principal.

\section{Los ambientes de creación}

Si se asume que todos los estudiantes tienen la potencialidad para ser creadores respecto a determinado contenido, entonces es necesario considerar que cuando esta potencialidad no se realiza, se debe a un conjunto de causas, una de las cuales puede ser el efecto entorpecedor del ambiente donde supuestamente ha de tener lugar la creación. Los ambientes de creación han sido bastante estudiados en la literatura sobre el tema, especialmente en los dedicados al aprendizaje grupal y las organizaciones.
El ambiente de creación es una construcción social que incluye las influencias de un momento y lugar determinados, del medio físico, las interacciones humanas, las representaciones de los sujetos y los significados; al igual que los objetos, productos y estados subjetivos de los miembros de determinada comunidad o grupo. Es la integración peculiar de estos factores, que se constituye como elemento facilitador o entorpecedor del desarrollo creativo.

En general existe consenso en torno a que un ambiente entorpecedor plantea restricciones al libre fluir del pensamiento, en él las ideas que surgen, las iniciativas de los sujetos, se coartan al estar sometidas a criterios valorativos en etapas tempranas de su surgimiento. Si bien parece conveniente no pensar siempre de manera tan pesimista (Labarrere y Quintanilla, 1999), es cierto que los intentos por el desarrollo de la creatividad, han tomado cuenta de este hecho y la mayoría de ellos tratan de situar a la persona en ambientes con un mínimo de restricciones o «totalmente» libre de ellas; o combinar la libertad de producción, con restricciones, juicios, etc., nunca presentes en las primeras etapas (consideradas como verdaderamente creativas). En este sentido, el modelo clásico aparece en el empleo que se hace en la enseñanza de técnicas para la creación, como el Brainstorming, la Sinéctica y otras.

En el ámbito escolar el ambiente ha sido tratado de manera muy similar a como lo ha sido en otros terrenos y, por ejemplo, muchas aproximaciones a la creatividad de los estudiantes son proclives a introducir el juego como vía de asegurar la necesaria libertad para crear. Se presupone que éste constituye el motor principal del desarrollo del sujeto creativo.

El efecto facilitador de las situaciones lúdicas es reconocido, pero no deja de ser cuestionable más allá de las primeras edades. El criticismo a interponer se relaciona con la naturaleza misma de la creatividad, infantil y adulta y con aspectos como la intención y dirección de la creatividad, tratados anteriormente (Rubinstein, 1967; Gardner, 1985).

Volviendo a los ambientes, en nuestras investigaciones diferenciamos ambientes entorpecedores y favorecedores del desarrollo del sujeto creativo, hemos procedido así a partir de estimar la inclusión activa consciente y dirigida al desarrollo. 


\section{LABARRERE}

Que el ambiente sea favorecedor, depende de un amplio espectro de factores interrelacionados, no hay un efecto lineal que pueda argumentarse, incluso suponiendo que los sujetos «coinciden en un mismo ambiente», hay que reconocer que para unos podría tener efectos favorecedores y actuar contrariamente respecto a otros (Labarrere y Quintanilla, 1999). Por esta razón, intentar una tipología de ambientes favorecedores, que actúen positivamente en todos los casos y sobre todos los sujetos, es bastante infructuoso. A lo más que uno puede aspirar es a ofrecer un conjunto de principios, o recomendaciones, para el trabajo con los ambientes.

Desde la concepción aquí sustentada y la investigación de fondo correspondiente (Labarrere, 1996; Quintanilla, 1997; Labarrere y Quintanilla, 1999; Carriera, 2000; Verde 2000, Jiménez, 2000 y Sánchez, 2000), los ambientes «facilitadores» del desarrollo deben cumplir con las siguientes propiedades:

1) debe ser problematizador

2) además altamente interactivo

3) debe ponderar la producción novedosa, actuar sobre la intencionalidad y la iniciativa creadora

4) y ser también denso en actos metacognitivos

Con estos parámetros nos hemos aproximado al estudio y desarrollo de la creatividad en la escuela y a los procesos de educación del sujeto creador. Cada uno de ellos se relaciona con dimensiones que permiten la aproximación a la creatividad como un hecho holístico.

El carácter problematizador se asocia a los procesos creativos y al acto de creación en sus mecanismos más íntimos: crear es avanzar hacia lo nuevo a través del planteamiento y la solución de problemas. Sabemos que el pensamiento creador arranca de situaciones donde a las personas les resulta imposible actuar con los medios (conocimientos, estrategias, etc.) que están directamente accesibles y se ven obligados a penetrar en espacios de actividad no habituales para ellas. Es en esos espacios donde se produce la novedad que caracteriza al acto creativo y sus productos y, por tanto, donde se genera la potencialidad requerida para la formación del sujeto creador.

$\mathrm{La}$ interactividad como característica y requisito del ambiente de creación, proviene de considerar a ésta como un hecho de comunica- ción entre los sujetos; de ver la creatividad desde lo grupal o colectivo y no sólo en su dimensión individual. La ponderación de lo novedoso responde a la necesidad de estimar la creatividad por sus productos. Por su parte, la necesaria acción sobre la intencionalidad e iniciativa creadoras son elementos básicos en el desarrollo del sujeto creativo en los planos individual y grupal y, por último, la densidad metacognitiva resulta un elemento básico, asociado al carácter consciente y reflexivo del sujeto.

Más recientemente, junto con Quintanilla (Labarrere y Quintanilla, 1999), comenzamos a trabajar la noción de ambiente restrictivo como factor de desarrollo de la creatividad. Con una aproximación operativa, por ambiente restrictivo estamos entendiendo el que potencialmente puede generarse cuando la persona es situada en condiciones que «conspiran contra su expresión creativa»; es decir, en situaciones donde no le resulta fácil evidenciar su creatividad y debe vencer ciertos obstáculos "sobreañadidos" y especialmente previstos desde la intencionalidad pedagógica. Dichos obstáculos, que funcionan como «barreras» para la creatividad personal, pueden generarse a dos niveles: 1) en el entorno físico material y 2) a nivel psicosocial.

Partimos del criterio según el cual el desarrollo de la creatividad se produce en contextos donde no siempre las condiciones creadas actúan a favor de las personas. Esta manera de ver las cosas se diferencia de aquellas en que la acción pedagógica sobre la creatividad como generación de condiciones que favorecen su desarrollo (ver por ejemplo Mitjans, 1998).

En el ambiente restrictivo, a la dificultad intrínseca generada por el carácter problemático de la situación se suma la que proviene de la «manipulación pedagógica» de las circunstancias; que resulta diferenciable de aquella que se genera desde la intencionalidad de que el sujeto opere en el "límite" de sus posibilidades creativas. Desde nuestro punto de vista, la conformación de ambientes restrictivos es apropiada en momentos tardíos del desarrollo de la creatividad del sujeto en contextos pedagógicos, es decir, donde la creatividad individual o grupal ya ha adquirido cierto grado de madurez; en este sentido, la acción sobre los ambientes y su conformación como restrictivos, presenta importancia capital para actuar sobre el fomento de la iniciativa personal del sujeto creador. 


\section{CREATIVIDAD, APRENDIZAJE CREATIVO Y DESARROLLO DEL SUJETO ...}

La relación de aspectos que acabamos de presentar no garantiza, sin más, la formación de un sujeto creativo, en realidad mucho depende de lo que la persona pueda y quiera hacer por sí misma. Tampoco se descuenta la influencia de otros factores «externos», como los concurrentes en la familia y otros grupos e instituciones sociales. Por otro lado, es evidente que cualquier intento por desarrollar el sujeto creativo, debe tener en cuenta los conocimientos y los instrumentos para actuar en un contexto determinado; sin esto no hay creatividad que valga ni sujeto creativo alguno. No se crea a partir, o acerca, de aquello que no se conoce, y para crear es necesario disponer de instrumentos adecuados.

Es muy posible que a nuestra revisión de tópicos tuviera que añadirse lo concerniente a los conocimientos específicos sobre las áreas particulares o específicas de creación, así como también ofrecer instrumentos pertinentes para actuar en ella. Con independencia de lo anterior, resulta de por sí importante enfocar aspectos como los revisados, los cuales sin duda requieren de un tratamiento más pormenorizado.

\section{Referencias}

Carriera, J. (1998) El ambiente para la creación: Una opción en la educación de la creatividad, Tesis de Magíster, La Habana.

Corral, R. (1999) En torno al concepto de zona de desarrollo próximo. Rev. Cubana de Psicología, La Habana.

Feuerstein, R. (1980) Instrumental Enrichment. Baltimore: University Park Press.

Gardner, H. (1995) Estructuras de la mente. Fondo de Cultura Económica, México.

González, F. (1997) Epistemología cualitativa y subjetividad. Pueblo y educación, La Habana.

González, F. y Mitjans, M. (1989) La personalidad, su educación y desarrollo. Pueblo y educación, La Habana.

Jiménez, C. (2000) La Metacognición y su significado para los procesos de resolución de problemas matemáticos en estudiantes de nivel medio superior. Tesis de Magíster, La Habana.

Labarrere, A. (1996) Inteligencia y Creatividad en la escuela, Rev. Educación No. 88, La Habana.

(1997) Aprendizaje ...que le oculta la enseñanza. Rev. Siglo XXI, No. 7, México.

(2000) Aprendizaje para el Desarrollo, Rev. Cubana de Psicología, No. 1, 10-18.

(2000) Inteligencia y creatividad en la escuela: Repensar algunos fundamentos. San Luis de Potosí, México.

Labarrere, A. y Quintanilla, M. (1999) La creatividad como proceso de desarrollo en ambientes restrictivos. Rev. Pensamiento Educativo, No. 25, Santiago de Chile, PUC.
Martínez, R. (2000) Los procesos reflexivos en la creatividad del profesor. Tesis de Doctorado, La Habana.

Mitjáns, A. (1996). Creatividad y Personalidad. Implicaciones metodológicas y educativas. Pueblo y Educación, La Habana.

-El Talento en la discapacidad. Revista Siglo XXI, México.

Quintanilla, M., Labarrere A. (2000) Análisis de textos producidos por profesores de ciencia en ejercicio, en un espacio teórico-reflexivo de perfeccionamiento continuo En: Boletín de Investigación Educacional, Vol. 15 del 2000, 369-387.

Rubinstein, S. (1986) Psicología general. Pueblo y Educación, La Habana.

Sánchez, D. (2000) Las creencias en la solución de problemas matemáticos: enfoque desde la reflexión del alumno. Tesis de Magíster, La Habana.

Torre, S. de la (1993) Aprender de los errores. España: Escuela Española.

co: Trillas.
(1996). Creatividad y formación. Méxi- 